High-efficiency diffractive $x$-ray optics from sectioned multilayers

H. C. Kang, G. B. Stephenson, C. Liu, R. Conley, A. T. Macrander, J. Maser, S. Bajt, H. N. Chapman

December 17, 2004

Applied Physics Letters 
This document was prepared as an account of work sponsored by an agency of the United States Government. Neither the United States Government nor the University of California nor any of their employees, makes any warranty, express or implied, or assumes any legal liability or responsibility for the accuracy, completeness, or usefulness of any information, apparatus, product, or process disclosed, or represents that its use would not infringe privately owned rights. Reference herein to any specific commercial product, process, or service by trade name, trademark, manufacturer, or otherwise, does not necessarily constitute or imply its endorsement, recommendation, or favoring by the United States Government or the University of California. The views and opinions of authors expressed herein do not necessarily state or reflect those of the United States Government or the University of California, and shall not be used for advertising or product endorsement purposes. 


\title{
High-efficiency diffractive $x$-ray optics from sectioned multilayers
}

\author{
H. C. Kang and G. B. Stephenson ${ }^{a)}$ \\ Materials Science Division, Argonne National Laboratory, Argonne, IL 60439 \\ C. Liu, R. Conley, A. T. Macrander, and J. Maser \\ Advanced Photon Source, Argonne National Laboratory, Argonne, IL 60439 \\ S. Bajt and H. N. Chapman \\ Lawrence Livermore National Laboratory, Livermore, CA 94550
}

\begin{abstract}
We investigate the diffraction properties of sectioned multilayers in Laue (transmission) geometry, at hard x-ray energies (9.5 and $19.5 \mathrm{keV}$ ). Two samples are studied, a 200 period W/Si multilayer of $29 \mathrm{~nm} d$-spacing, and a 2020 period Mo/Si multilayer of $7 \mathrm{~nm}$ $d$-spacing, with cross-section depths ranging from 2 to $17 \mu \mathrm{m}$. Rocking curves across the Bragg reflections exhibit well-defined interference fringes originating from the depth of the sample. Efficiencies as high as $70 \%$ were obtained. This exceeds the theoretical limit for standard zone plates operating in the multi-beam regime, demonstrating that all of the intensity can be directed into a single diffraction order in small-period structures.
\end{abstract}

PACS numbers :

\footnotetext{
a) Author to whom correspondence should be addressed ; electronic mail : stephenson@anl.gov
} 
Breakthroughs in efficient, high-resolution diffractive optics for hard x-rays (e.g. to focus to nanometer-scale spots [1], or to compress to femtosecond-scale pulses [2]) are technically challenging but theoretically very promising. High spatial resolution requires correspondingly thin structures transverse to the beam direction, while high efficiency requires a relatively large depth along the beam direction. Fabrication of such high-aspect-ratio nanostructures challenges the limits of lithographic techniques. An alternative approach is to make a cross-section of a multilayer film, as in "sputter/slice" zone plates [3-5]. Although very high aspect ratios can be produced, the challenge in this case is to deposit a multilayer with hundreds or thousands of accurately placed layers, and to maintain the structure during sectioning. The optical properties of sectioned multilayers in the Laue (transmission) geometry, also known as volume gratings, have been calculated and measured in the soft x-ray region [2,6-8], but are relatively unexplored in the hard x-ray region. In theory, the properties are very promising: as the layer thickness becomes smaller, the efficiency should approach unity because all of the intensity can be directed into a single diffraction order.

We have been exploring the fabrication and hard x-ray diffraction properties of sectioned multilayers in Laue geometry. Results have been obtained for two multilayers of differing period and composition that bridge the gap between large and small layer thickness behavior. In a preliminary publication [9], we reported x-ray rocking curves showing fringes that allow us to determine the depth of the sections. Here we report measurements of the peak reflectivity as a function section depth, which demonstrate that high efficiency can be obtained at small periods. We find reasonable agreement between measurements and dynamical diffraction theory, providing a solid foundation for both the design and fabrication of novel hard x-ray optics. 
Two constant-period multilayers were grown on $\mathrm{Si}$ (001) substrates by DC magnetron sputter deposition techniques, as described elsewhere $[9,10]$. Multilayer $d$ spacings and layer volume fractions were determined by modeling Bragg-geometry xray reflectivity data. Multilayer A is a 200 -period W/Si multilayer with a $d$-spacing of $29 \mathrm{~nm}$ and $\mathrm{W}$ volume fraction of $60 \%$, with a total thickness of $6 \mu \mathrm{m}$. Multilayer B is a 2020-period Mo/Si multilayer with $d$-spacing of $7 \mathrm{~nm}$ and Mo volume fraction of $50 \%$, with a total thickness of $14 \mu \mathrm{m}$. These may be the thickest $\mathrm{x}$-ray-optical-quality multilayer films produced to date. As the outermost zone of a focusing structure, the 7 $\mathrm{nm}$ period of Multilayer B would correspond to a $4.2 \mathrm{~nm}$ diffraction-limited focal size [1].

To study the Laue-geometry diffraction behavior as a function of section depth, we produced cross sections by sectioning and polishing with depths intentionally varying by $\sim 10 \mu \mathrm{m}$ across a $2 \mathrm{~mm}$ length so that a single sample could be used to study a range of depths. The Laue diffraction geometry used is shown in Fig. 1 (inset). Here we define $z$ as the layer stacking direction ("thickness"), $x$ perpendicular to a cross-sectioned surface ("depth"), and y along the wedge ("length"). The x-ray scattering was mapped by scanning the scattering vector in the $Q_{x}$ and $Q_{z}$ directions. The dependence on section depth was determined by translating the wedge-shaped sample along the $y$ direction. The incident beam illuminated $50 \mu \mathrm{m}$ of the sample along the $y$ direction. Incident beams larger than the sample in the $z$ direction were used for sample angle scans to minimize effects of the center of rotation misalignment. Measurements of absolute reflectivity at the Bragg peak using carefully-aligned incident beams equal in size to the sample thickness were used to correct the intensity scale of the angle scans. Measurements were performed at beamline 12BM of the Advanced Photon Source. 
Using a $\operatorname{Si}(111)$ monochromator, an x-ray energy $E$ of either 9.5 or $19.5 \mathrm{keV}(\lambda=1.31$ or $0.64 \AA$ ) was selected.

Figure 1 shows a typical radial scan along $Q_{z}$ in the specular direction $\left(Q_{x}=0\right)$. Bragg reflections from the fundamental multilayer $d$-spacing and higher harmonics are observed. The pattern is symmetric about zero, which illustrates a feature of the unusual Laue geometry: diffraction from the "top" or "bottom" of the multilayer is equivalent. Figure 2 shows typical transverse (rocking) scans along $Q_{x}$ at the first-order Bragg reflection in $Q_{z}$, for $E=9.5 \mathrm{keV}$ and various values of section depth. The scattering patterns exhibit interference fringes around the central peak. The section depth $w$ of the illuminated region of the wedge can be obtained from the period $\Delta Q_{x}$ of the interference fringes using $w=2 \pi / \Delta Q_{x}$. The large number of fringes indicates that the sectioning process produced smooth truncation of the multilayer with a well-defined depth. The central part of the profile displays a complex dependence on $w$. For small $w$, the profiles have the shape of classical Fraunhofer single-slit diffraction patterns [11]. As $w$ increases, the intensity of the central maximum decreases relative to those of the fringe maxima. The intensity at $Q_{x}=0$ becomes a local minimum at $w \approx 8$ or $15 \mu \mathrm{m}$ for multilayer A or B, respectively. At larger values of $w$, the intensity at $Q_{x}=0$ becomes a local maximum again.

Figure 3 shows the reflectivity of the first-order Bragg peak at $Q_{x}=0$ and $E=9.5$ $\mathrm{keV}$, as a function of $w$. Quite high absolute reflectivities are obtained, near $70 \%$ for multilayer B at the optimum depth. The second-order peak is also shown for multilayer $\mathrm{A}$; it is very weak for multilayer $\mathrm{B}$, because of the equal thicknesses of the Mo and $\mathrm{Si}$ layers. The oscillatory behavior observed with increasing $w$ corresponds to the pendellösung phenomenon that occurs in dynamical diffraction from crystals in Laue 
geometry [12]. Observation of these oscillations verifies the conceptual basis of dynamical diffraction theory for multilayers in the hard x-ray region, and indicates that the sectioned multilayer samples are of sufficient structural quality to produce dynamical effects.

An analytical solution for the reflectivity of small-period multilayers can be developed by extending the 2-beam dynamical theory for crystals. For a centrosymmetric crystal in the symmetric Laue geometry, in the limit of small diffraction angle, the reflectivity is given by $[12,13]$

$$
R=\left.\exp \left[\frac{2 \pi w \operatorname{Im}\left(\psi_{0}\right)}{\lambda}\right]\left|\sin \left(\frac{\pi w \psi_{H}}{\lambda} \sqrt{1+\eta^{2}}\right)\right|\right|^{2}\left|1+\eta^{2}\right|^{-1},
$$

where $\eta$ is related to the transverse wavenumber $Q_{x}$ by $\eta=Q_{x} \lambda / 2 \pi \psi_{H}$. For a binary multilayer, the normalized structure factors in the forward and diffracted-beam directions, $\psi_{0}$ and $\psi_{H}$, are given by $\psi_{H}=2 \Delta n \sin (\pi L x) / \pi L, \quad \psi_{0}=2(\langle n\rangle-1)$. Here $n$ is the refractive index, \langle\rangle represents the mean of the two layers in the multilayer, e.g. $\langle n\rangle=$ $x n_{1}+(1-x) n_{2}, \Delta$ represents the difference between the two layers, e.g. $\Delta n=n_{1}-n_{2}, x$ is the volume fraction of layer 1 , and $L$ is the order of the $00 L$ Bragg peak. The refractive indices of each the layers are complex numbers given by $n=1-\delta$ - i $\beta$, where $\delta$ and $\beta$ depend on material and photon energy [14]. Note that the reflectivity is independent of the $d$-spacing. At the center of the rocking curve, where $\eta=0$, the specular reflectivity as a function of section depth $w$ can be expressed as

$$
R=\exp \left[-\frac{4 \pi w\langle\beta\rangle}{\lambda}\right]\left(\sin ^{2}\left[\frac{2 w \sin (\pi L x) \Delta \delta}{\lambda L}\right]+\sinh ^{2}\left[\frac{2 w \sin (\pi L x) \Delta \beta}{\lambda L}\right]\right) .
$$

The $\sin ^{2}$ term gives the oscillatory pendellösung component, with a period $\Delta w=$ $\pi \lambda L|2 \Delta \delta \sin (\pi L x)|^{-1}$, while the $\sinh ^{2}$ term gives the anomalous transmission (Borrmann effect) for samples with large section depth [12]. The pendellösung period depends on 
$\Delta \delta$, and is thus a direct measure of the scattering contrast of the multilayer.

Dashed curves in Fig. 3 show reflectivities calculated using the 2-beam expression, Eq. (2), with standard elemental densities for the W, Mo, and Si layers. For the firstorder peaks, there is qualitative agreement with the observed depth dependence, while the predicted oscillation period with depth of the second-order peak for multilayer $\mathrm{A}$ is significantly larger than observed. It is interesting to consider the predicted dependence of the first-order pendellösung period on sample composition and x-ray wavelength obtained from the 2-beam expression. For a multilayer with equal thickness layers $(x=$ 0.5 ), the period for $L=1$ is given by $\Delta w=\pi \lambda / 2 \Delta \delta$. (This is a factor of $\pi / 2$ larger than for a phase zone plate.) Ignoring anomalous scattering effects near absorption edges, the real part of the refractive index can be expressed using $\delta=r_{e} \rho_{e} \lambda^{2} / 2 \pi$, where $r_{e}=2.82 \times$ $10^{-13} \mathrm{~cm}$ is the Thomson radius of the electron and $\rho_{e}$ is the electron density [15]. This gives a first-order pendellösung period of $\Delta w=\pi^{2} / r_{e} \Delta \rho_{e} \lambda$, which is inversely proportional to the density difference between the layers and to photon wavelength. We confirmed this wavelength dependence, finding that the depth giving maximum reflectivity increased by approximately a factor of two when the wavelength was decreased from 1.31 to $0.64 \AA$. The observed period for multilayer B in Fig. 3(c) is $\sim 20 \%$ larger than predicted, which may indicate that the density difference in the sample is lower than that between pure Mo and $\mathrm{Si}$, owing to interdiffusion and/or reaction to form $\mathrm{MoSi}_{\mathrm{x}}$ [16]. The relatively shallow minimum in the observed reflectivity from multilayer $\mathrm{B}$ at larger depths is likely due to the finite transverse resolution of the measurement, which can be seen in Fig. 2(b).

Effects of scattering into multiple directions are not included in the 2-beam solution. One such effect can be seen in the rocking curves in Fig. 2. For multilayer B, the 
features at $Q_{x}= \pm 0.0017 \AA^{-1}$ occur when either the incident or exit beam makes the Bragg angle with the fundamental $L= \pm 1$ reflection. For multilayer A, this condition occurs at $Q_{x}= \pm 0.0001 \AA^{-1}$, so that the features are part of the complex shape of the central peak. Multi-beam effects contribute to the specular reflectivity whenever the section depth is small enough to broaden the specular peak by an amount comparable to the spacing between adjacent Bragg peaks. Conversely, the 2-beam expression (2) is valid and multi-beam effects are negligible for the $L=1$ peak when the sample depth $w$ is large enough so that the rocking width of the $L=1$ peak, $\mid \Delta \theta \cong d / 2 w$, is smaller than its distance from the $L=2$ peak, $\Delta \theta_{B} \cong \lambda / 2 d$, where $d$ is the multilayer period. This condition can be written as

$$
d^{2}<\lambda w
$$

which is satisfied for multilayer B, but not always for multilayer A, in this study. For thicknesses above this limit, multiple diffraction orders are simultaneously excited, so that the efficiency into any one order is limited. For example, the maximum theoretical efficiency is $\sim 40 \%$ for an ideal binary zone plate in the multi-beam regime [17]. For thicknesses satisfying relation (3), all of the intensity can be directed into one diffraction order by choice of incidence angle, giving a peak reflectivity near unity for hard x-rays with low absorption. Substituting the $x=0.5$ expression for optimum sample depth $w=$ $\Delta w / 2$ into condition (3) cancels the wavelength dependence, giving $d<\pi /\left(2 r_{e} \Delta \rho_{e}\right)^{1 / 2}$. Thus the condition depends only on the multilayer materials. For Mo/Si and W/Si, the limit occurs at $d=30$ and $21 \mathrm{~nm}$, respectively. Multi-beam effects occur only away from the center of the rocking curve for $7 \mathrm{~nm} \mathrm{Mo/Si} \mathrm{multilayer} \mathrm{B}$, but at the center of the rocking curve for $29 \mathrm{~nm}$ W/Si multilayer A.

To model multi-beam scattering, we have carried out calculations using a version of 
coupled-wave $(\mathrm{CW})$ theory $[18,19]$. The relationship between the complex dielectric constants used in CW theory and the optical constants used in the 2-beam theory is derived from the relation $n=\varepsilon^{1 / 2}$, and can be expressed as $\Delta \varepsilon \approx 2 \Delta \delta-2 i \Delta \beta$. We have numerically solved the CW theory for the reflectivity of sectioned multilayers using a $2^{\text {nd }}$ order Runge Kutta algorithm [20]. A total of 60 eigenmodes was used to describe the wavefield inside the sections. This provides convergence of the solutions in both the 2beam and multi-beam regimes. Reflectivity results from n-beam CW theory are displayed as solid curves in Fig. 3. For multilayer B, the multi-beam solution differs little from the 2-beam solution. For multilayer A, the difference is large. The lower peak reflectivity and more complex functional form of the multi-beam solution for the firstorder peak agree better with the experimental results, as do the positions of the maxima and minima for the second-order peak. The differences between the experimental data and the multi-beam theory could be due to interfacial roughness and experimental resolution not included in the calculation.

In summary, using deposition and sectioning of multilayer films, we have successfully fabricated volume gratings that give high reflectivities for hard $\mathrm{x}$-rays in the Laue geometry. These structures have the high ratio of section depth to layer thickness (e.g. 2000 at the reflectivity maximum for multilayer B) needed to produce efficient hard x-ray optics with sub-10-nm spatial resolution. We observe oscillatory reflectivity as a function of section depth, with high diffraction efficiency at small periods in agreement with dynamical diffraction theory. This provides a promising demonstration of the techniques and principles for the design of novel hard x-ray optics using sectioned multilayers.

The authors would like to thank the BESSRC beamline staff for capable assistance. 
Work performed under the auspices of the U.S. Department of Energy by ANL under contract W-31-109-ENG-38 and by UC, LLNL under contract W-7405-ENG-48. One of the authors (H.C.K.) also acknowledges support from the KOSEF postdoctoral fellowship program. 


\section{REFERENCES}

[1] J. Maser, G. B. Stephenson, S. Vogt, W. Yun, A. T. Macrander, H. C. Kang, C. Liu, and R. Conley, Proc. SPIE 5539, 185 (2004).

[2] H. N. Chapman and S. Bajt, unpublished.

[3] R.M. Bionta, K.M. Skulina, and J. Weinberg, Appl. Phys. Lett. 64, 945 (1994).

[4] B. Kaulich, Ph. D. thesis, University of Goettingen, Germany (1996).

[5] N. Kamijo, Y. Suzuki, H. Takano, S. Tamura, M. Yasumoto, A. Takeuchi, and M. Awaji, Rev. Sci. Instrum. 74, 5101 (2003); S. Tamura, M. Yasumoto, N. Kamijo, Y. Suzuki, M. Awaji, A. Takeuchi, H. Takano, and K. Handa, J. Synchrotron Radiation 9, 154 (2002).

[6] V. E. Levashov and A.V. Vinogradov, Appl. Opt. 32, 1130 (1993).

[7] D. Hambach, G. Schneider, and E.M. Gullikson, Opt. Lett. 26, 1200 (2001).

[8] J. Maser and G. Schmahl, Opt. Commun. 89, 355 (1992).

[9] H. C. Kang, G. B. Stephenson, C. Liu, R. Conley, A. T. Macrander, J. Maser, S. Bajt, and H. N. Chapman, Proc. SPIE 5537, 127 (2004).

[10] C. Liu, R. Conley, A. T. Macrander, J. Maser, H. C. Kang, M. Zurbuchen, and G. B. Stephenson, to appear in J. Vac. Sci. Technol. A (2005).

[11] M. Born and E. Wolf, Principles of Optics $6^{\text {th }}$ ed. (Pergamon, New York, 1980).

[12] B.W. Batterman and H. Cole, Rev. Mod. Phys. 36, 681 (1964).

[13] W.H. Zachariasen, Theory of X-ray Diffraction in Crystals (Constable and Co., London, 1945), p. 113.

[14] B.L. Henke, E.M. Gullikson, and J.C. Davis, At. Data Nucl. Data Tables 54, 181 (1993).

[15] R. W. James, The Optical Principles of the Diffraction of X-rays (G. Bell and Sons, 
London, 1948), p. 54.

[16] D. Stearns, R. S. Rosen, and S. P. Vernon, Proc. SPIE 1547, 2 (1991).

[17] J. Kirz, J. Opt. Soc. Am. 64, 301 (1974).

[18] R.Magnusson and T.K.Gaylord. J. Opt. Soc. Am. 68, 1777 (1978).

[19] R. R. A. Syms, Practical Volume Holography (Clarendon Press, Oxford, 1990).

[20] Numerical Algorithms Group, NAG Fortran Library, Mark 18 (1997). 


\section{FIGURE CAPTIONS}

Fig. 1. Typical radial scan for multilayer $B$, showing positive and negative orders of Bragg peaks $(E=19.5 \mathrm{keV}, w=10 \mu \mathrm{m})$. Inset: Schematic of Laue geometry used in this study.

Fig. 2. Measured transverse profiles (rocking curves) through the 001 reflection for various section depths $(E=9.5 \mathrm{keV})$. Profiles are offset by factors of 10 for clarity.

Fig. 3. Points: measured reflectivity of the 001 and 002 reflections as a function of section depth $(E=9.5 \mathrm{keV})$. Curves: calculations using 2-beam (dashed) and multibeam (solid) dynamical diffraction theory for the Laue geometry. 


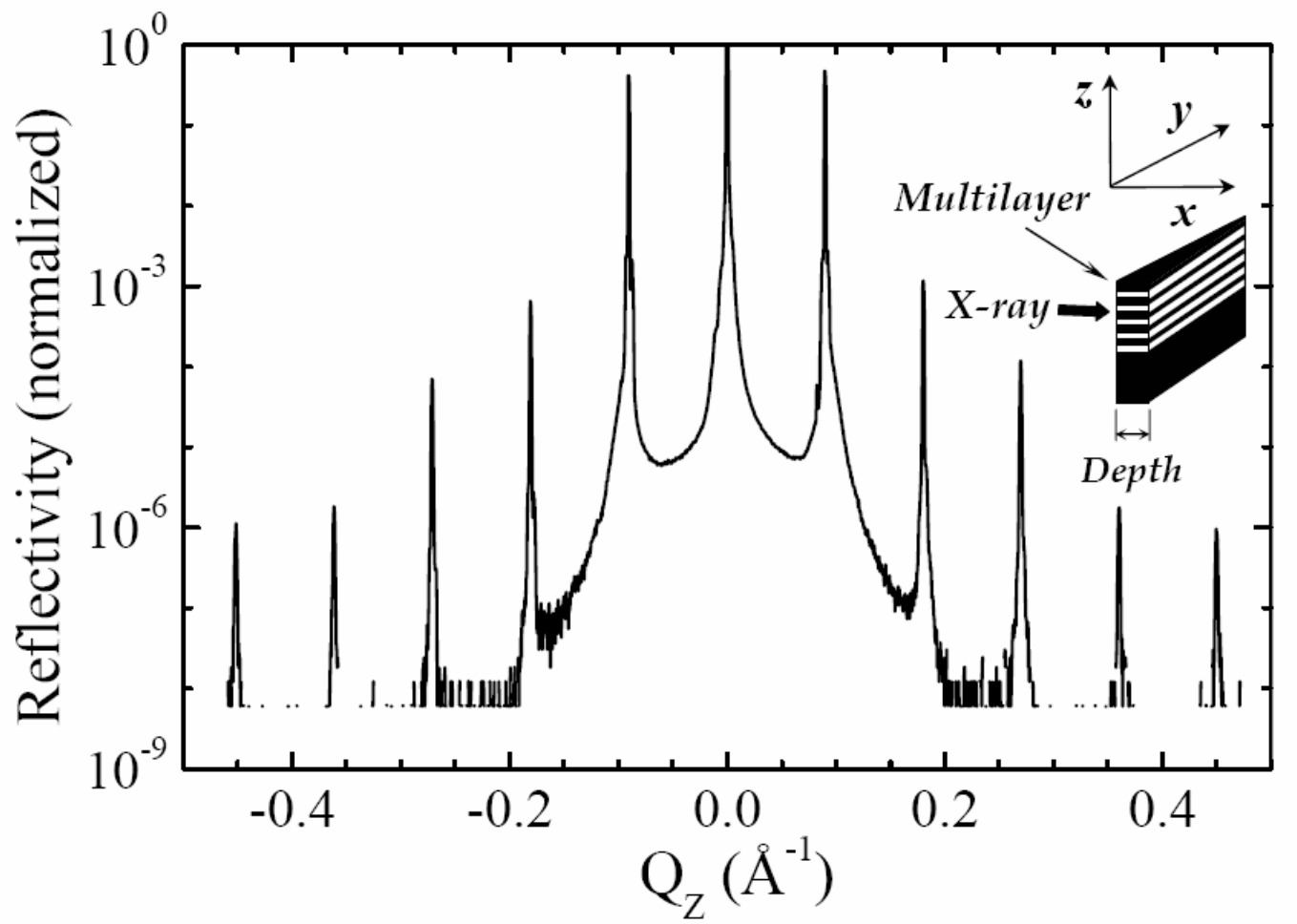

Fig. 1 of 3. Appl. Phys. Lett. H. C. Kang et al. 


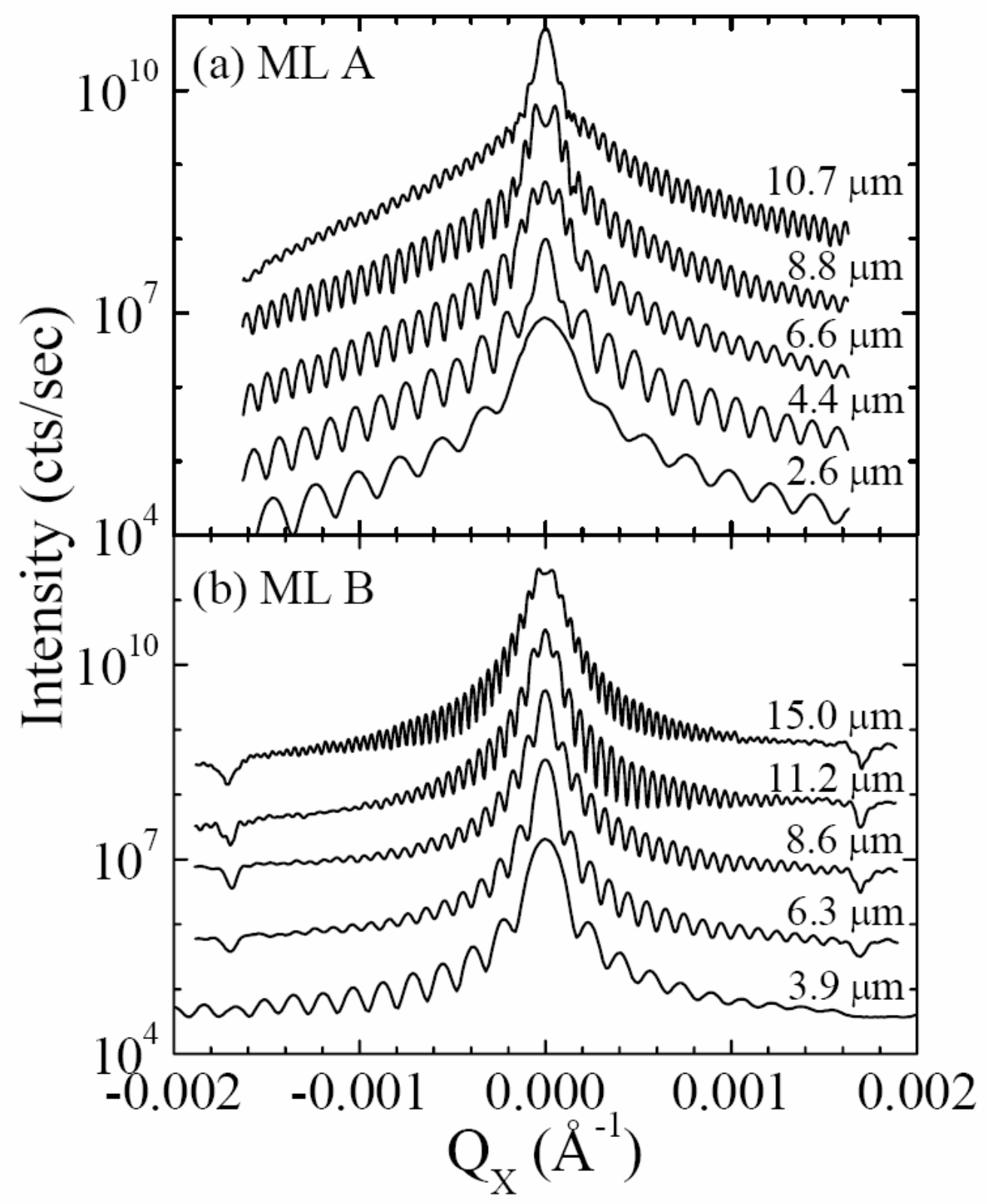

Fig. 2 of 3. Appl. Phys. Lett. H. C. Kang et al. 


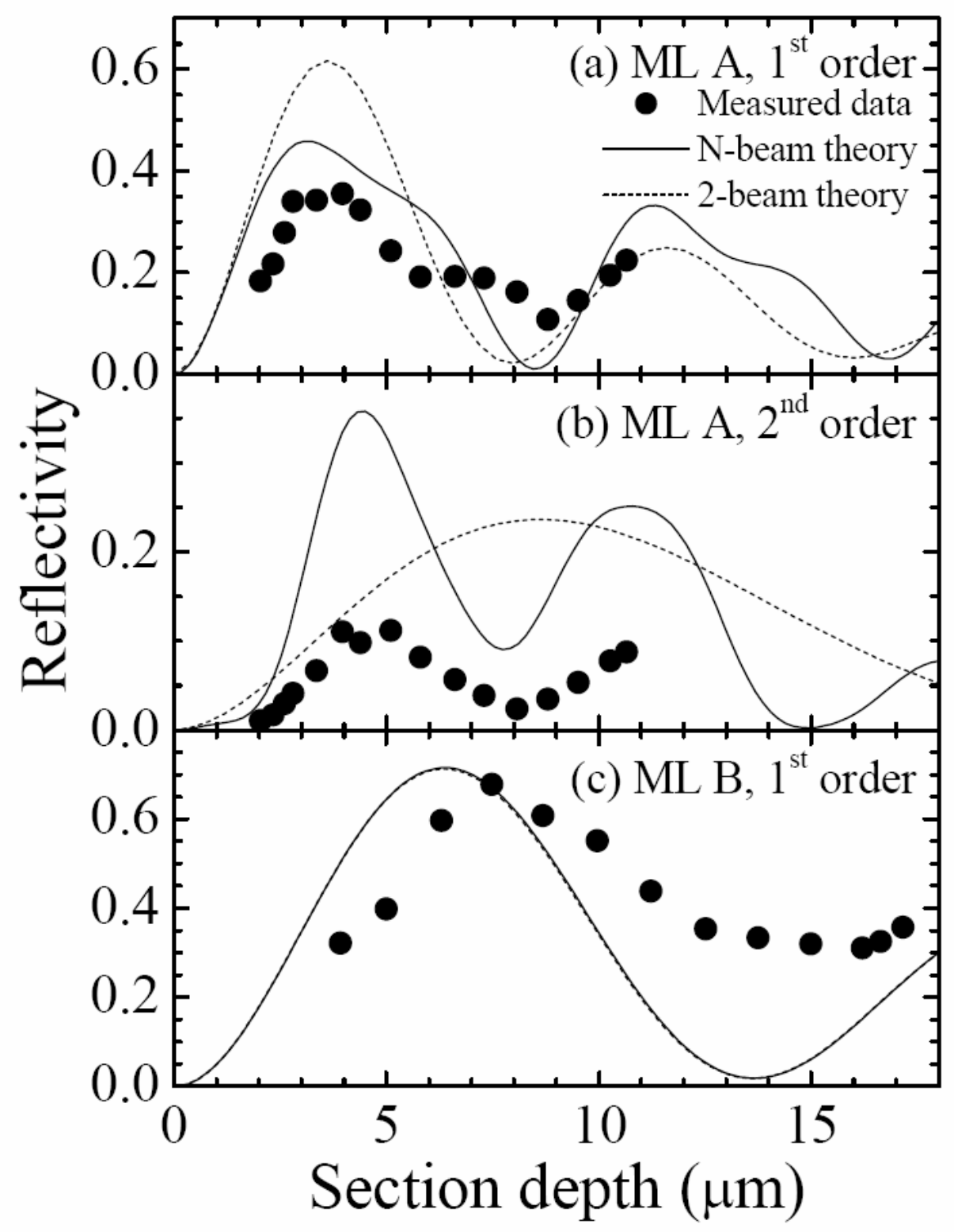

Fig. 3 of 3. Appl. Phys. Lett. H. C. Kang et al. 